Case Report

\title{
Chronic Pain Two Cures
}

James David Adams, Jr *

University of Southern California, School of Pharmacy, Los Angeles, CA, USA; E-Mail: jadams@usc.edu

* Correspondence: James David Adams; E-Mail: jadams@usc.edu

Academic Editor: Gerhard Litscher

Special Issue: Complementary and Alternative Medicine in Nervous System Conditions

OBM Integrative and Complementary Medicine 2018 , volume 3 , issue 4

doi:10.21926/obm.icm.1804035
Received: January 14, 2018

Accepted: December 14, 2018

Published: December 20, 2018

\begin{abstract}
:
Background: This study presents several case reports of chronic pain patients treated with two different plant medicines from the Chumash Indian tradition.

Methods: Patients suffering from self-reported fibromyalgia, chronic back pain, chronic bursitis, chronic tendinitis and other conditions were treated. California sagebrush (Artemisia californica) was grown by the author and made into a liniment. The liniment was applied to painful sites on the skin. Black sage (Salvia mellifera) was made into a sun tea. Chronic pain and other pain patients soaked their feet in the preparation.

Results: The liniment provided relief from even severe pain within a minute or so. After daily use for several days, chronic pain was resolved in several patients. The sun tea provided pain relief throughout the body within 20 minutes. After $1-7$ days of once daily use, several chronic pain patients had improved conditions.
\end{abstract}

Conclusions: Both A. californica liniment and S. mellifera sun tea provide pain medicines that according to patients, can cure chronic pain. Cure means the pain does not return.

\section{Keywords}

Chronic pain; cure; California sagebrush; black sage; monoterpenoids; sesquiterpenoids; pain chemokine cycle

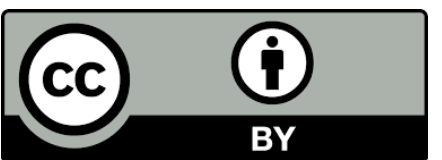

(C) 2018 by the author. This is an open access article distributed under the conditions of the Creative Commons by Attribution License, which permits unrestricted use, distribution, and reproduction in any medium or format, provided the original work is correctly cited. 


\section{Introduction}

Chronic pain affects $11 \%$ of US adults and increases with age [1]. There is no FDA approved cure for chronic pain. Chronic pain patients are treated with oral non-steroidal anti-inflammatory agents and oral opioids that kill over 100,000 patients every year from ulcers, heart attacks, strokes, seizures and respiratory depression [2].

Pain is sensed in the skin and is best treated in the skin [3, 4]. Transient receptor potential cation channels are abundant on sensory afferent neurons of the skin. These channels are the main pain receptors in the body. There are 28 different transient receptor potential cation channels that exist on nonoverlapping populations of skin sensory afferent neurons. These channels can be inhibited by plant derived monoterpenoids. It is critical to use a mixture of several different monoterpenoids to inhibit as many types of transient receptor potential cation channels as possible.

Chronic pain is caused by a cyclic, self-perpetuating mechanism in which stressed or damaged skin cells release chemokines that stimulate production of prostaglandins and IL-17 [2, 5]. Prostaglandins and IL-17 then stimulate chemokine production. Prostaglandins come from cyclooxygenase- 2 that is present in macrophages attracted into the skin by chemokines. Prostaglandins cause pain by interacting with prostaglandin receptors and enhance pain by inducing the phosphorylation of transient receptor potential cation channels. Skin resident T cells produce IL-17. This is the pain chemokine cycle. Oral medications do not adequately penetrate into the skin to inhibit these mechanisms and stop chronic pain.

The A. californica liniment has been successfully used on patients suffering from broken bones, pain from large abrasions, bruises, sprains, strains, spinal stenosis, osteoarthritis, rheumatoid arthritis, cancer pain, bursitis, muscle pain from over exertion, gout, hip replacement, knee replacement, headaches, migraine headaches, basilar migraines, diabetic neuropathy, shingles, bee stings, a gunshot wound to the abdomen and other conditions [6-8]. The S. mellifera sun tea has been successfully used to treat pain from abrasions, bruises, sprains, strains, osteoarthritis, rheumatoid arthritis, bursitis, muscle pain from over exertion, headaches and other conditions [6, 9].

\section{Materials and Methods}

The liniment was made as follows: $86 \mathrm{~g}$ of leaves and branches of A. californica, 1 leaf of Salvia apiana, one Persea americana seed and $500 \mathrm{ml}$ of $70 \%$ isopropanol were put into a brown glass jar for 6 weeks [7]. The plant material was removed leaving a dark green liniment. Sun tea was made from S. mellifera: $115 \mathrm{~g}$ of leaves and stems, 1 leaf of Salvia apiana, 2 liters of water were combined and placed in the sun for $4-6$ hours [6]. The plant material was removed leaving a redbrown sun tea.

The author is not a medical doctor and did not perform any diagnostics tests or examinations on any patient. The diagnoses provided to the patients by other medical professionals were used in this work. Many patients declined to provide information on family history, medications, other medical conditions, psychosocial history, or genetics. The data that was provided by patients is 
presented in the results. The author did not perform examinations or diagnoses after treatment, but relied on the ability of each patient to rate their pain. Many patients were treated once, reported pain relief, but were lost to follow up. These patients are not included in this work.

Chronic pain patients were given the liniment and instructed to apply it liberally to the skin at painful sites with a cotton ball or by spray. They were instructed to continue using the liniment once or twice daily for $5-7$ days. Other chronic pain patients were asked to soak their feet in the sun tea for 20 minutes or more. They were instructed to soak their feet in the sun tea once daily for 5 - 7 days. All chronic pain patients were asked to stop all oral pain medications, including opioids.

Before and after the initial treatment, patients were asked to rate their pain on a scale of $0-10$. They were asked to report their pain levels after $5-7$ days of treatment. Patients were recruited from the general population around Southern California. These were people who attended talks about Chumash Healing given by the author. Some of the patients attended because they were willing to try anything to treat their chronic pain, others attended in order to prove that the chronic pain treatments were ineffective. The patients did not share common beliefs or opinions. All patient personal information is protected and will not be published. The author is a recognized traditional healer in the Chumash Indian tradition. He is allowed to practice traditional healing and is protected by California State Law.

\section{Results}

Chronic pain has been successfully treated in many patients, at least 31 , with the liniment and/or the sun tea. It was noted with several patients that in order to improve chronic pain, all opioids had to be stopped, including fentanyl patches. This involved cutting opioid doses in half every week until all opioids were no longer used. Opioid induced hyperalgesia was noted in several patients. Chronic pain was resolved in many patients with back, neck and other pain.

A Caucasian man, 58 years old, suffered from chronic back pain and had a diagnosis of slipped disks. He began using the liniment and reported that he was cured of his chronic pain within 3 weeks. He no longer needed any pain medication and returned to a normal life. He had no other illnesses, was not using any medications and had no family history of chronic pain.

Another Caucasian man, 55 years old, suffered from chronic back pain and found that the liniment relieved his pain. He was reluctant to stop his oral opioids and struggled with his opioids for several weeks until he was able to stop them, while continuing the liniment. He then reported he was cured of his chronic pain. He did not recall if there was a family history of chronic pain and declined to discuss other medications or conditions he had.

A Caucasian woman, 48 years old, suffered from chronic back pain for many years. After using the liniment for three weeks and gradually stopping her opioids, she reported that her pain was much better and she no longer needed pain medicines. She had no family history of chronic pain and did not discuss other drugs or conditions she had.

A Caucasian man, 34 years old, suffered from chronic back pain for many years. He began using the liniment while gradually stopping his opioids and reported that he was cured of his pain after a month. He declined to discuss other details of his history or conditions. 
A Caucasian woman, 36 years old, suffered from chronic back pain, started using the liniment, gradually stopped using oral opioids and reported that she was cured after four weeks. She did not want to discuss other details of her history or medications.

A Latino man, 48 years old, suffered from a herniated disc since he was a child. He used the liniment for four weeks, gradually stopped his opioids and reported that he was cured of his pain. He did not suffer from other conditions, did not discuss other medications and had no family history of chronic pain.

A Caucasian man, 49 years old, suffered from chronic back pain due to spinal stenosis and reported pain relief when he used the liniment. After using the liniment for five weeks while gradually stopping oral opioids, he reported that he was cured of his chronic pain. He declined to discuss other details of his history or medications.

A young Caucasian woman, 25 years old, suffered from chronic back pain for several months after excessive gym exercises. She used the liniment while gradually stopping oral opioids over a three week period and reported that she was cured of her chronic pain. She had no family history of chronic pain, was not using other medications and was otherwise healthy.

A young Caucasian woman, 26 years old, suffered from chronic back pain after a car accident. She used the liniment for four weeks while gradually stopping oral opioids and reported she was cured of her pain. She had no family history of chronic pain, did not suffer from other conditions and was not using other medications.

A Caucasian man, 28 years old, suffered from chronic back pain after a car accident. He treated his pain daily with six tablets of oxycodone. He reported that the liniment relieved his pain. He slowly weaned himself off of oxycodone and continued the liniment. One week after stopping the oxycodone, he reported that he was cured of his chronic pain and no longer needed pain medication. He was otherwise healthy, had no family history of chronic pain and used no other medications.

An Asian woman, 53 years old, suffered from chronic back pain for several years. After using the liniment for one month while stopping oral opioids, she reported she was cured of her pain. She declined to discuss other details of her history or health.

A pregnant, woman 32 years old, suffered from chronic back pain. She used the liniment for one week and reported that her back pain was gone and did not return. She was otherwise healthy, had no family history of chronic pain and was not taking other medications.

A 70 year old Caucasian man suffered from chronic back pain for a year. He used the liniment several times, which relieved his pain and reported that his pain did not return. He declined to discuss other details of his history or health.

A 29 year old Asian man suffered from chronic back pain and found that the liniment helped tremendously. His pain did not come back. He was otherwise healthy, had no family history of chronic pain and was not using other medications.

A 62 year old Caucasian man had suffered from chronic back pain for several years. He tried the liniment and said it did not relieve his pain. He tried the liniment a few more times over the next few days and said he got no relief from his pain. This patient declined to say if he was using opioids and declined to discuss other details of his condition or history.

A male Asian, 72 years old, had suffered from chronic back pain for several years. He used the sun tea for one week and reported that he was cured of his pain. He declined to discuss other details of his history or health. 
A Caucasian woman, 73 years old, suffered from postsurgical chronic back pain, used the sun tea and the liniment for one week and reported she was cured of her pain. She suffered from no other conditions, was not using other medications and did not report her family history.

A Caucasian woman, 75 years old, suffered from chronic back pain for 15 years. She used the sun tea and the liniment for one week and reported she was cured of her pain. She did not suffer from any other condition and declined to report her history or other medications.

A Caucasian woman, 63 years old, suffered from fibromyalgia for many years. Her fibromyalgia limited her ability to do her job. She used the sun tea for one week and the liniment for one month, while stopping all other pain medications, and reported she was cured of her fibromyalgia pain. She was otherwise healthy, did not use other medications and had no family history of chronic pain.

A Caucasian woman, 75 years old, suffered from chronic shoulder pain for many years. She used the liniment for one week and reported she was cured of her chronic pain. She was in the practice of using marijuana regularly, did not use other medications, had no family history of chronic pain and suffered from hip arthritis.

A Caucasian woman, 64 years old, suffered from chronic shoulder pain for several months. She used the liniment for five days and reported that she was cured of her pain. She did not suffer from other conditions, had no family history of chronic pain and did not discuss the use of other medications.

A Caucasian man, 61 years old, suffered from chronic bursitis of the neck, shoulders and back for several months. He used the liniment for one week and reported he was cured of his bursitis pain. He was otherwise healthy, used no other medications and had a family history of chronic pain in his mother.

A Caucasian man, 55 years old, suffered for several years from bursitis in the right shoulder that interfered with sleep. He was addicted to oxycodone that was used to treat his pain. The liniment cured his bursitis pain temporarily, only as long as he could avoid taking oxycodone. Oxycodone induced hyperalgesia was a prominent problem in this patient. When he started oxycodone again, the pain returned. He eventually stopped taking oxycodone and reported the liniment cured his chronic pain. He declined to discuss other details of his health or history.

A Caucasian woman, 64 years old suffered from chronic shoulder pain for several months. She used the liniment for one week and reported she was cured of her pain. She was otherwise healthy and declined to discuss the use of other medications or details of her health or history.

A Caucasian man, 60 years old, suffered from tendinitis in his right leg for several months. He reported that his pain was cured after using the liniment for one week. He was otherwise healthy and declined to report other details of his history or health.

A Latino woman, 78 years old, suffered from tendinitis in her right leg for several years. She used the liniment for one week and reported that she was cured of her pain. She declined to discuss other details of her health or history.

A Caucasian man, 42 years old, suffered from chronic knee pain for several months. He used the liniment for one week and reported that he was cured of his pain. He was otherwise healthy and did not discuss other details of his health or history.

A Caucasian woman, 28 years old, suffered from chronic foot pain. She used the liniment for one week and reported that she was cured of her pain. She did not suffer from other conditions, did not use other medications and had no family history of chronic pain. 
A young Caucasian man, 26 years old, suffered from chronic knee pain. He used the liniment and reported he was cured of his pain after one week. He was otherwise healthy and did not discuss other details of his health or history.

A Caucasian man, 32 years old, suffered from chronic knee and shoulder pain. He used the liniment for one week and reported he was cured of his pain. He was otherwise healthy, did not use any medications and had no family history of chronic pain.

A Caucasian man, 27 years old, suffered from chronic knee pain for 3 months. He used the liniment one time and reported that he was cured of his chronic pain. He was healthy in every other way, did not use any medications and had no family history of chronic pain.

A Latino woman 58 years old suffered from chronic pain in her left hip for several months. She applied the liniment to her hip and received rapid relief of her pain. She continued to use the liniment daily for one week and reported that the pain did not return. She did not discuss other details of her health or history.

Many chronic pain patients were treated once with the liniment or the sun tea, reported relief of their pain but did not respond to requests for follow up information. It is not known if their chronic pain improved more than one day.

\subsection{Limitations of the Study}

The current work was conducted by a Pharmacologist who is a Traditional Healer, not a medical doctor. Therefore, medical records were not available. Many patients declined to state the cause of their pain or their family histories. Few patients were willing to discuss their drug therapy or other illnesses. The study was not placebo controlled. The investigator and the patients were not blinded to the identity of the drug used.

\section{Discussion}

The author applied for an Investigational New Drug application to the Food and Drug Administration in 2011 in order to perform a clinical trial with the liniment. The application was denied because the reviewing officials were not convinced of the safety of the liniment. The liniment is a traditional Chumash Indian remedy that has been used by the author and other practitioners of Chumash Healing in several hundred patients with no safety concerns or reports of tolerance, addiction or adverse drug events. In July of 2018, the Food and Drug Administration denied the New Drug application from the author for black sage sun tea, stating that more treatment data and safety data must be provided. The sun tea is a traditional medicine used by the Chumash Indians of California. The author has used black sage sun tea in many patients. None of the patients in the current study reported tolerance, addiction or adverse effects from the liniment or sun tea.

Several patients reported that they were able to stop using opioids because they found pain relief from the liniment or sun tea. Some of the patients were addicted to opioids and struggled to overcome their addictions. Most of the patients using opioids said that the liniment or sun tea helped them overcome their addiction and in some cases, opioid induced hyperalgesia.

One of the great fallacies in medicine is that pain comes from the brain and other internal sites. Therefore, pain must be treated in the brain with oral or injected medicines. The current report treated pain only in the skin, and provides evidence of improvements in chronic pain. The current 
report confirms that the safest and most effective way to treat pain is in the skin (2-8). Of course, the brain and brain stem are involved in processing pain.

Is the pain relief reported here due to the placebo effect? It is possible that some of the pain relief was due to the placebo effect, or perhaps due to the charisma of the author. However, in the author's experience, chronic pain patients tend to be hostile toward charismatic people who offer placebos. It may be possible that a placebo controlled clinical trial could elucidate the placebo effects of the medicines used in this study. As stated before, the author has applied to the FDA to perform placebo controlled, double blind, randomized clinical trials of the medicines described in this work. The FDA has declined to approve the clinical trials.

Chronic pain patients were taught how to make the liniment and sun tea themselves for free. Many patients make the liniment for themselves and their acquaintances. The plants used to make the medicines are common, not endangered and can be grown in gardens to make harvesting easier.

The liniment and the sun tea contain several monoterpenoids that stop pain by inhibiting transient receptor potential cation channels and cure chronic pain by inhibiting the synthesis of IL17 (2). This stops the pain chemokine cycle. Monoterpenoids are largely cleared from the skin by evaporation, only a small percentage passes through the skin and is cleared by the liver and kidneys.

The liniment contains sesquiterpenoids that penetrate into the skin and down regulate cyclooxygenase-2 (5). This shuts down the synthesis of prostaglandins and stops the pain chemokine cycle of chronic pain. Oral medications do not penetrate adequately into the skin to stop chronic pain. Sesquiterpenoids are largely cleared from the skin by evaporation. Only a small percentage is cleared by the liver and kidneys.

\section{Conclusions}

The Chumash Indians of California teach us how to cure chronic pain. The current case study provides the reason to perform randomized, double blind, placebo controlled clinical trials of the liniment and the sun tea. In the meantime, practitioners of Chumash Healing will continue to treat and resolve chronic pain.

\section{Acknowledgements}

The author gratefully acknowledges the Chumash Indians of California for teaching him how to treat pain and resolve chronic pain.

\section{Author Contributions}

The author performed all the duties described and wrote the manuscript.

\section{Funding Source}

The author has not been able to get any funding from any source to perform this work. 


\section{Competing Interests}

The author has no competing interests.

\section{Additional Materials}

Table 1 Summary of chronic pain patients treated in this study. DTS - declined to state.

\begin{tabular}{|c|c|c|c|c|c|}
\hline Age & $\begin{array}{l}\text { Site of chronic } \\
\text { pain }\end{array}$ & $\begin{array}{l}\text { Family history } \\
\text { of pain }\end{array}$ & $\begin{array}{l}\text { Duration of } \\
\text { pain }\end{array}$ & $\begin{array}{l}\text { Duration of } \\
\text { pain relief }\end{array}$ & $\begin{array}{l}\text { Adverse } \\
\text { reactions }\end{array}$ \\
\hline 58 & Back & no & $>1$ year & $>3$ weeks & none \\
\hline 55 & Back & DTS & Months & >weeks & none \\
\hline 48 & Back & no & Years & $>3$ weeks & none \\
\hline 34 & Back & DTS & Years & $>4$ weeks & none \\
\hline 36 & Back & DTS & DTS & $>4$ weeks & none \\
\hline 48 & Back & no & Years & $>4$ weeks & none \\
\hline 49 & Back & DTS & DTS & $>5$ weeks & none \\
\hline 25 & Back & no & Months & $>3$ weeks & none \\
\hline 26 & Back & no & Months & $>4$ weeks & none \\
\hline 28 & Back & no & Months & $>4$ weeks & none \\
\hline 53 & Back & DTS & Years & $>4$ weeks & none \\
\hline 32 & Back & no & 2 months & $>1$ week & none \\
\hline 70 & Back & DTS & 1 year & $>1$ week & none \\
\hline 29 & Back & no & DTS & $>1$ week & none \\
\hline 62 & Back & DTS & Years & none & none \\
\hline 72 & Back & DTS & Years & $>1$ week & none \\
\hline 73 & Back & DTS & Months & $>1$ week & none \\
\hline 75 & Back & DTS & $>15$ years & $>1$ week & none \\
\hline 63 & Fibromyalgia & no & Years & $>1$ month & none \\
\hline 75 & Shoulder & no & Years & $>1$ week & none \\
\hline 64 & Shoulder & No & Months & $>5$ days & none \\
\hline 61 & Shoulder & yes & Months & $>1$ week & none \\
\hline 55 & Shoulder & DTS & Years & $>1$ month & none \\
\hline 64 & Shoulder & DTS & Months & $>1$ week & none \\
\hline 60 & Leg & DTS & Months & $>1$ week & none \\
\hline 78 & Leg & DTS & Years & $>1$ week & none \\
\hline 42 & Knee & DTS & Months & $>1$ week & none \\
\hline 28 & Foot & no & DTS & $>1$ week & none \\
\hline 26 & Knee & DTS & DTS & $>1$ week & none \\
\hline 32 & Knee and shoulder & no & DTS & $>1$ week & none \\
\hline 27 & Knee & no & 3 months & $>1$ day & none \\
\hline 58 & Hip & DTS & Months & >1 week & none \\
\hline
\end{tabular}




\section{References}

1. Nahin R. Estimates of pain prevalence and severity in adults: United States, 2012. J Pain. 2015; 16: 769-780.

2. Adams J. Chronic pain can it be cured? J Pharmaceut Drug Devl. 2017; 4: 105-109.

3. Adams J, Wong X. Control of pain with topical plant medicines. Asian Pacific J Tropical Biomed. 2015; 5: 930-935.

4. Adams J. The effects of yin, yang and chi in the skin on pain. Medicines. 2015; 3: 1-8.

5. Adams J, Haworth I, Coricello A, Perri F, Nguyen C, Aiello F, et al. The treatment of pain with topical sesquiterpenes. Front Nat Prod Chem. 2017; 3: 176-195.

6. Garcia C, Adams J. Healing with medicinal plants of the West - Cultural and scientific basis for their use. 3rd ed revised. La Crescenta: Abedus Press, 2016.

7. Fontaine $\mathrm{P}$, Wong V, Williams T, Garcia C, Adams J. Chemical composition and antinociceptive activity of California sagebrush (Artemisia californica). J Pharmacog Phytother. 2013; 5: 1-11.

8. Adams J. The use of California sagebrush (Artemisia californica) liniment to control pain. Pharmaceuticals. 2012; 5: 1045-1053. doi:10.3390/ph5101045.

9. Adams J, Garcia C. The advantages of traditional Chumash healing. Evidence Based Comp Alt Med. 2005; 2: 19-23. PMCID: PMC1062165

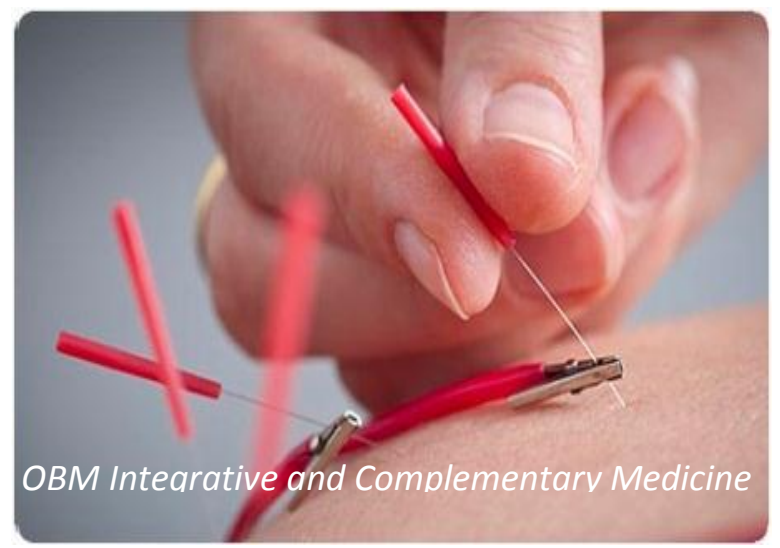

Enjoy OBM Integrative and Complementary Medicine by:

1. Submitting a manuscript

2. Joining in volunteer reviewer bank

3. Joining Editorial Board

4. Guest editing a special issue

For more details, please visit: http://www.lidsen.com/journals/icm 